\title{
LA REVISTA DE MEDICINA Y CINE COMO PLATAFORMA PARA FOMENTAR EL APRENDIZAJE DE LA PSICOLOGÍA
}

\section{The Journal of Medicine and Movies as a platform to promote learning in Psychology}

Jorge PÉREZ

Grupo de Investigación Educativa en Ciencias de la Salud (GRECS). Universidad Pompeu Fabra. Barcelona (España). Autor para correspondencia: Jorge Pérez.

Correo electrónico: jordi.perez@upf.edu

Recibido: 9 de junio de 2020

Aceptado: 15 de junio de 2020

Ante la invitación de uno de los editores de la revista, aceptamos el reto de reflexionar sobre las posibilidades que tiene la Revista de Medicina y Cine (RMC) en la formación de estudiantes de Psicología, una importante rama de las ciencias de la salud.

La Psicología es definida como la ciencia que estudia el comportamiento humano y, por tanto, no nos tendría que extrañar que cualquier película fuera susceptible de estudio psicológico ya que en todas ellas aparece la conducta humana. No solo nos referimos a películas dramáticas para ilustrar aspectos relacionados con la Psicopatología, quizá lo más conocido, sino con cualquiera de los géneros clásicos del cine. Por citar algunos ejemplos de películas que adoramos, el western Centauros del desierto (1956) de John Ford sería adecuado para ilustrar la ira o la compasión; el film bélico El puente sobre el rio Kwai (1957) de David Lean podría mostrar el fenómeno psicológico del síndrome de Estocolmo; el film de romanos Espartaco (1960) de Stanley Kubrick serviría perfectamente para debatir sobre el odio, el amor y la solidaridad; el musical Cantando bajo la Iluvia (1952) de Stanley Donen y Gene Kelly presenta escenas de envidia y amor; la comedia El apartamento (1960) de Billy Wilder, nuestra la importancia de los roles sociales y la obediencia, 


\section{LA REVISTA DE MEDICINA Y CINE COMO PLATAFORMA PARA FOMENTAR \\ EL APRENDIZAJE DE LA PSICOLOGÍA \\ JORGE PÉREZ}

y la película de dibujos animados Up (2009) de Pete Docter y Bob Peterson sería un buen instrumento para reflexionar sobre el amor de pareja o el comportamiento altruista.

En una revisión, creemos exhaustiva, de los artículos publicados hasta el momento en la RMC en relación con la Psicología, hemos encontrado 15 documentos: siete de ellos $^{1-7}$ aparecieron en un número monográfico sobre Psicoanálisis en 2008; cuatro artículos analizan una película concreta para reflexionar sobre la eutanasia ${ }^{8}$, el acoso laboral ${ }^{9}$ o diferentes tipos de psicopatología ${ }^{10,11}$; uno diserta sobre las patologías a través del cine $\mathrm{e}^{12}$; otro muestra una selección de películas para ilustrar el tema de la discapacidad física ${ }^{13}$; otro reflexiona sobre la memoria a partir de un capítulo de una serie ${ }^{14} ; y$, finalmente, otro muestra una experiencia docente sobre el uso de la literatura para favorecer el aprendizaje de las patologías psicológicas ${ }^{15}$.

Desde casi nuestros inicios en la docencia universitaria hemos utilizado las películas comerciales como soporte para fomentar el aprendizaje de los estudiantes universitarios. Si bien lo hemos hecho en materias de introducción a la biología ${ }^{16}$ o de bioética ${ }^{17}$, fundamentalmente ha sido en asignaturas de Psicología.

En nuestros primeros años en la Facultad de Medicina de la Universidad Autónoma de Barcelona (UAB), usamos diversas películas para reflexionar sobre temas específicos: Recuerda (1945) de Alfred Hitchcock sobre el psicoanálisis, Frances (1982) de Graeme Clifford referente a las terapias biomédicas invasivas como la lobotomía o Charly (1968) de Ralph Nelson para ilustrar el desarrollo de la inteligencia humana. Posteriormente, también en la $\cup A B$, utilizamos tres películas como elemento clave para realizar evaluaciones formativas: El milagro de Ana Sullivan (1962) de Arthur Penn, Alguien voló sobre el nido del cuco (1975) de Milos Forman y La Naranja Mecánica (1971) de Stanley Kubrick.

En la Facultad de Ciencias de la Salud y de la Vida en la Universidad Pompeu Fabra (UPF) seguimos utilizando el cine, de uso frecuente en dicho centro ${ }^{18}$, en dos materias relacionadas con las ciencias del comportamiento en la extinta licenciatura de Biología. En la asignatura de Psicobiología, en el tercer curso, colaboramos con la profesora responsable en el uso de tres películas para debatir sobre temas concretos del programa: Rain man (1988) de Barry Levinson referente al autismo, A propósito de Henry (1991) de Mike Nichols sobre los tipos y problemas de la memoria y Una mente maravillosa (2001) de Ron Howard en relación con la esquizofrenia. En la asignatura de Psicología Humana, en cuarto curso y de la que éramos los responsables, usamos las ya citadas El milagro de Ana Sullivan y La naranja mecánica para realizar evaluaciones formativas.

Ya con la instauración del Espacio Europeo de Educación Superior (EEES), las anteriores dos películas siguieron siendo usadas, con el mismo fin, para fomentar el aprendizaje de la psicología en tres asignaturas de tres grados diferentes de la UPF: Criminología, Biología Humana y Medicina.

Como puede apreciar el lector hemos tenido una gran experiencia en el uso del cine para favorecer el aprendizaje de la Psicología de cuyos resultados nos sentimos orgullosos. Creemos que debe haber numerosos profesores de Psicología que utilicen el cine como herramienta docente, es por ello que pensamos que la RMC puede ser una buena plataforma para que éstos expongan sus experiencias.

Hace algún tiempo habíamos argumentado que la RMC, además de colaborar en su formación, podía servir para fomentar la adquisición de la competencia transversal de escribir artículos científicos en estudiantes de Ciencias de la Salud ${ }^{19,20}$. También la RMC debería servir a los estudiantes de Psicología.

Así, invitamos a docentes y alumnos universitarios de carreras relacionadas con la psicología que envíen sus experiencias sobre el uso del cine, o de otros recursos humanísticos, en la mejora de la docencia o del aprendizaje. 


\section{LA REVISTA DE MEDICINA Y CINE COMO PLATAFORMA PARA FOMENTAR \\ EL APRENDIZAJE DE LA PSICOLOGÍA \\ JORGE PÉREZ}

\section{Referencias}

1. José Elías García Sánchez, Enrique García Sánchez. Medicina y cine: diferentes caras del análisis de una relación. Rev Med Cine [Internet] 2008; 4(1): 1.

2. Sangro Colón P, Huerta Floriano MA. Cine y psicoanálisis: la fábrica de sueños. Rev Med Cine [internet] 2008; 4(1):2-3.

3. Sangro Colon P. El cine en el diván: teoría fílmica y psicoanálisis. Rev Med Cine [internet2008; 4(1): 4-11.

4. Pérez X. El psicoanalista a través de sus traumas. Rev Med Cine [internet] 2008; 4(1): 12-6.

5. Huerta Floriano MA. El cine como terapia: el psicoanálisis en la obra de Woody Allen. Rev Med Cine [Internet] 2008; 4(1): 17-26.

6. Sánchez Noriega JL. La cultura psicoanalítica en el cine negro americano. Rev Med Cine [Internet] 2008: 4(1): 27-34.

7. Huerta Floriano MA, Sangro Colón P. Psicología: Freud, pasión secreta. El padre del psicoanálisis. Rev Med Cine [Internet] 2008; 4(1): 35-7.

8. Aguado Díaz A-L, González González M, Rozada Rodríguez C, Alcedo Rodríguez MA. Mar adentro (2004). Una visión diferente seis años después del Óscar. Rev Med Cine [Internet] 2010; 6(2): 40-6.

9. Vizoso-Gómez CM. En tierra de hombres (2005). El acoso psicológico en el trabajo. Rev Med Cine [Internet] 2016; 12(3): 163-9.

10. Bocic M, Vargas A. Rio Místico / Mystic River (2003): "Hay hilos en nuestras vidas. Si uno tira de ellos, todo lo demás se ve afectado». Rev Med Cine [Internet] 2019; 15(2): 67-71.

11. Viana-Vivanco E, Flores-Pereira AA, Alvarez-Baeza A, Janssen-Aguilar R, Méndez-Domínguez NI. La baraja del Joker: factores desencadenantes de la conducta agresiva en el paciente con afección pseudobulbar. Rev Med Cine [Internet] 2020; 16(3): 165-74.

12. Vera Poseck B. Locura y cine: claves para entender una historia de amor reñido. Rev Med Cine [Internet] 2006; 2(3): 80-8.

13. Monjas Casares MI, Arranz Moro F. El cine como recurso para el conocimiento de las personas con discapacidad: Veinticinco películas de la última década. Rev Med Cine [Internet] 2010; 6(2): 55-68.

14. Salomé Lima N. Memoria perpetua. Comentario del episodio "The entire history of you» de "Black Mirror (2011)». Rev Med Cine [Internet] 2015; 11(3): 147-56.

15. Torres Montesinos C, Macas Ordóñez EF. Práctica de innovación docente: lee y despatologízate. Rev Med Cine [Internet] 2018; 15(1): 45-8.

16. Pérez J. El uso de la película Despertares (1990) para evaluar aprendizajes en la licenciatura de Biología de la Universidad Pompeu Fabra. Diez años de experiencia. Rev Med Cine [Internet] 2009; 5(1): 27-9.

17. Farré M, Pérez J. Uso del cine comercial en la docencia de Bioética en estudios de Biología. Rev Med Cine [Internet] 2011; 7(1): 3-7.

18. Pérez J, Aramburu, J, Baños JE, Bosch F, Díez J, Faré $M$, Girvent $M$, Sentí $M$, Valverde $O$. Uso del cine comercial como herramienta docente en estudios en ciencias de la salud. Una experiencia multidisciplinar y colectiva. FEM 2014, 17 (3): 131-5.

19. Pérez J. La Revista de Medicina y Cine, un medio para fomentar el aprendizaje de estudiantes en ciencias de la Salud. Rev Med Cine [Internet] 2013; 9(4): 149-50.

20. Pérez J, Fresnadillo MJ, García-Sánchez JE, GarcíaSánchez E. La Revista de Medicina y Cine como facilitador de publicaciones científicas de los estudiantes. Rev Med Cine [Internet] 2020; 16(1): 43-5. 


\section{LA REVISTA DE MEDICINA Y CINE COMO PLATAFORMA PARA FOMENTAR \\ EL APRENDIZAJE DE LA PSICOLOGÍA \\ JORGE PÉREZ}

\begin{tabular}{|l|l|} 
& $\begin{array}{l}\text { Jorge Pérez Sánchez. Es profesor titular jubilado de Psicología Médica y } \\
\text { miembro del Grupo de Investigación Educativa en Ciencias de la Salud } \\
\text { (GRECS) de la Universidad Pompeu Fabra (UPF). Fue Director de la Oficina } \\
\text { Educativa (1998-2016) y Decano (1999-2011) de la Facultad de Ciencias de } \\
\text { la Salud y de la Vida de la UPF. Recibió el premio a la trayectoria docente del } \\
\text { Consejo Social de la UPF en } 2006 \text { y la distinción Vicens Vives de la Generalitat } \\
\text { de Catalunya a la calidad docente en } 2011 .\end{array}$
\end{tabular}

